\title{
DIFICULDADES E FACILIDADES DOS DOENTES NO SEGUIMENTO DO TRATAMENTO DA HIPERTENSÃO ARTERIAL
}

\section{DIFFICULTIES AND FACILITIES OF THE PATIENTS IN FOLLOWING THE TREATMENT OF ARTERIAL HYPERTENSION}

Valdênia Dias de Castro* Marcia Regina Car**

CASTRO, V.D.; CAR, M.R. Dificuldades e facilidades dos doentes no seguimento do tratamento da hipertensão arterial. Rev.Esc.Enf.USP, v.33, n.3, p.294-304, set. 1999.

\begin{abstract}
RESUMO
Este estudo teve por objetivos identificar as dificuldades e as facilidades de doentes no seguimento do tratamento da hipertensão arterial; e, verificar se existem diferenças nessas dificuldades e facilidades em dois momentos dessa trajetória - no atendimen to ambulatorial e na internação. Para este estudo descritivo, quantitativo, foram entrevistados 34 doentes, com diagnóstico médico de hipertensão arterial (HA) elou suas complicações, sendo 17 matriculados no ambulatório e 17 hospitalizados na unidade de internação de nefrologia. Os resultados revelaram que, em geral, não houve diferenças entre as dificuldades e as facilidades referidas pelos doentes para o seguimento do tratamento da HA, nos dois momentos estudados. As dificuldades e facilidades apontadas referem-se ao financiamento do tratamento (compra de medicamento e gastos com transporte); acessibilidade ao serviço (facilidade em marcar consulta médica e distância do serviço de saúde); mudança de hábito alimentar (dieta hipossódica) e a importância do apoio familiar nesta trajetória.
\end{abstract}

UNITERMOS: Pressão arterial. Hipertensão.

\begin{abstract}
This study aims to identify difficulties and facilities of the patients in following the treatment of the arterial hypertension; and, to verify differences that exist between those difficulties and facilities in two moments of the trajectory - in the ambulatorial treatment and in the hospital stay. For this quantitative, descriptive study, 34 patients with medical diagnosis of arterial hypertension or its complications were interviewed, being 17 registered in the ambulatorial unit and 17 hospitalized in the nefrology unit. The results of the study revealed that, in general, there were no differences among the difficulties and the facilities referred by the patients for the following of the treatment in the two studied moments. The difficulties and facilities pointed out by the patients refer to the financing of the treatment (medication purchase and expenses with transport); accessibility to the service (easiness in marking medical consultation and distance of the service of heakth); change of alimentary habit (hipossodic diet) and the importance of the family support in this trajectory.
\end{abstract}

UNITERMS: Blood pressure. Hypertension.

\section{INTRODUÇÃO}

O controle da hipertensão arterial, na prevenção de riscos para as doenças cardiovasculares e renais, envolve a complexidade do seguimento do seu tratamento. As condições de pressão arterial (PA) elevada trazem conseqüências a longo prazo para o organismo, ou seja, quanto maior a PA (sistólica ou diastólica) maior é a ocorrência de complicações nos órgãos-alvo: cérebro, coração, visão, vasos, rins (BRASIL, 1993a).

* Enfermeira. Instituto de Infectologia Emílio Ribas.

** Enfermeira. Professora Doutora do Departamento de Enfermagem Médico-Cirúrgica da Escola de Enfermagem da USP 
A HA e o rim interagem de forma intima $e$ complexa podendo a primeira ser causa ou conseqüência de doença renal. Um defeito na função renal quase certamente está associado na patogenia da hipertensão essencial (primária), enquanto que a insuficiência renal crônica (IRC) é a causa mais comum de hipertensão arterial secundária. Portanto, "há uma correlação entre o nível de PA e a progressão da insuficiência renal. A incidência e a intensidade da hipertensão aumentam progressivamente à medida que a função renal deteriora, de forma que todos os pacientes com IRC estão hipertensos quando iniciam o tratamento dialítico" (PASCOAL; MION JR., 1996).

Segundo THOMÉ (1994), as doenças renais são menos incidentes do que outros problemas de saúde pública no Brasil, porém os custos sócio-econômicos são elevados. Entretanto, em nosso país são poucos os estudos sobre formas de prevenção para essas doenças, uma vez que havendo prevenção e controle para a HA provavelmente reverte-se a agressão renal.

Dentre os fatores de risco para as doenças cardiovasculares, a HA é um dos mais importantes, afetando cerca de 11 a $20 \%$ da população entre idade acima de 20 anos (BRASIL, 1988). Estima-se que no Brasil cerca de $15 \%$ da população adulta possa ser rotulada como hipertensa, aumentando-se a prevalência a medida que a idade avança. A HA "considerada um dos principais fatores de risco de morbidade e mortalidade cardiovasculares, seu alto custo social é responsável por cerca de $40 \%$ dos casos de aposentadoria precoce e de absenteísmo no trabalho em nosso meio". O controle adequado dessa situação reduz significativamente os riscos individuais e os custos sociais" (CONSENSO, 1998).

Estudos patrocinados pelo Banco Mundial e pela OMS encontraram resultados que dão uma idéia de com a hipertensão está representada no mundo. Evidenciou-se maior sobrecarga para os países subdesenvolvidos, já que sua população é apenas um terço da população total. "Das 50 milhões de mortes estimadas no mundo para 1990 , quase 3 milhões, ou $5,8 \%$ poderiam ser atribuídas à $\mathrm{HA}$, variando este percentual de 1 a $11 \%$ ficando a América Latina e o Caribe em torno de 8,1\%" (ACHUTTI; ACHUTTI, 1997). Segundo COSTA (1994), "indivíduos de raça negra têm maior prevalência de hipertensão, sofrem mais dano renal por HAS e tem deterioração mais rápida da função renal".

Por outro lado, a grande maioria desses indivíduos ignoram ser hipertensos. Portanto, não são atendidos pelo Sistema de Saúde para essa doença. Entretanto, daqueles que sabem ser hipertensos, a maioria abandona o tratamento por vários motivos, e aqueles que fazem tratamento não são atendidos adequadamente, aumentando, assim, o risco para as complicações cardíacas, cerebrais e renais. Estas complicações diminuem a qualidade e a expectativa de vida (BRASIL, 1988).

A adesão do paciente hipertenso, portanto, é fundamental para se obter o controle da pressão arterial, porém, ainda constitui um grande desafio para a equipe de saúde, visto as altas taxas de abandono (cerca de 30 a 50\%), principalmente entre os assintomáticos. São inúmeros os fatores que determinam os baixos índices de adesão, apesar da eficácia dos medicamentos no controle dos níveis pressoricos. Estes fatores estão relacionados, principalmente, à doença, ao medicamento, aos pacientes e ao médico (DELITTI; SILVA, 1996; PIERIN et al, 1984; BLACK; MATASSARIN JACOBS, 1993).

Outra questão importante para a eficácia do tratamento é a acessibilidade ao serviço de saúde. Segundo FLEURY; GIOVANELLA (1996), "acessibilidade, além da disponibilidade de recursos de atenção à saúde em determinado local e tempo, compreende as características dos recursos que facilitam ou dificultam o seu uso por parte dos clientes. Ou seja, é o grau de ajuste entre as características dos recursos de atenção à saúde e as da população no processo de busca e obtenção da atenção". Ou melhor dizendo, é a capacidade de um grupo para buscar e obter atenção.

Portanto, segundo os autores referidos acima, acesso é um conjunto de fatores adequados entre o cliente e o sistema de saúde, tais como: disponibilidade, acessibilidade, acomodação, capacidade financeira e aceitabilidade. Assim, os serviços de saúde recebem influências dos seguintes fatores: sócio-culturais, fatores relacionados ao consumidor, fatores relacionados ao prestador de serviço; fatores organizacionais; características da estrutura; acessibilidade geográfica (distância, tempo, custo da viagem); processo de prestação de cuidado.

Outro conceito considera acessibilidade como "uma noção complexa, integrada pelas categorias de: distância/tempo entre a população e as unidades de saúde; oportunidade, entendida como noção de prestar serviço em sintonia com a demanda; funcionalidade, vista com a capacidade dos serviços para resolver os problemas; custos, incluindo não apenas os custos diretos da atenção mas também os indiretos como medicamentos, salário perdido, preço do transporte; e aceitação do demandante do modo e características como se produz a atenção" (FLEURY; GIOVANELLA, 1996).

O seguimento do tratamento para o controle da hipertensão arterial e fundamental na prevenção de riscos cardiovasculares (RIBEIRO; ZANELLA; KOHEMANN JR., 1996) e de piora progressiva da 
função renal (CARVALHO, 1997). Segundo TOTO et al (1995); PASCOAL; MION JR. (1996), o controle da pressão arterial está associado ao retardo da progressão global da insuficiência renal.

Considerando-se assim a importância e a complexidade do seguimento do tratamento da HA, este estudo teve por objetivos:

- identificar as dificuldades e as facilidades de doentes, de unidade de internação e em atendimento ambulatorial, no seguimento do tratamento da hipertensão arterial; e,

- verificar se existem diferenças nas dificuldades e facilidades de doentes, de unidade de internação e em atendimento ambulatorial, no seguimento do tratamento da hipertensão arterial.

\section{PERCURSO METODOLÓGICO}

Este é um estudo descritivo, exploratorio, quantitativo sobre o seguimento do tratamento da hipertensão arterial por sujeitos hipertensos. Seguindo os critérios de inclusão dos hipertensos propostos neste estudo, foram entrevistados 34 doentes, sendo 17 deles matriculados no ambulatório de nefrologia e 17 internados na unidade de nefrologia do Hospital das Clínicas da Faculdade de Medicina da Universidade de São Paulo. A técnica de amostragem utilizada neste estudo foi a não probabilística de acordo com a disponibilidade dos pesquisadores no período de coleta de dados.

Critérios de inclusão dos hipertensos no estudo:

- doentes adultos com diagnóstico médico de hipertensão arterial, submetidos a tratamento ambulatorial, nos meses de setembro e outubro de 1997, que não tenham tido necessidade de internação hospitalar pela doença e suas complicações;
- doentes adultos com diagnóstico médico de hipertensão arterial, submetidos a tratamento na unidade hospitalar de internação, nos meses de setembro e outubro de 1997, devido à doença ou suas complicações.

A coleta de dados seguiu as seguintes etapas:

1. solicitação de consentimento da instituição para a realização da pesquisa;

2. seleção dos doentes compatíveis com os critérios de inclusão no estudo;

3. obtenção de consentimento informado de cada doente para sua participação no estudo;

4. aplicação de formulário de entrevista (Anexo).

Este formulário inclui duas partes distintas. A primeira constituída de dados de identificação, de condições sócio-econômicas e diagnóstico médico. A segunda parte envolveu dados sobre o seguimento do tratamento da HA desde a descoberta do diagnóstico e as orientações recebidas, até as dificuldades e facilidades apontadas pelos doentes para esse seguimento. Após a realização das entrevistas, os dados foram inseridos no programa dBase IV plus e analisando em software EpiInfo 5.0. Assim, os dados serão apresentados, no item 3 a seguir, utilizando tabelas com números absolutos e percentuais.

\section{APRESENTAÇÃO E DISCUSSÃO DOS RESULTADOS}

Na tabela abaixo (Tabela 1) verificou-se um predomínio de doentes do sexo feminino $(64,7 \%)$ no ambulatório e masculino na unidade de internação $(58,8 \%)$. Observou-se que a maioria dos hipertensos estudados têm idade acima de 41 anos.

Tabela 1 - Distribuição dos doentes, por unidades de tratamento, quanto ao sexo e a idade. São Paulo, 1997.

\begin{tabular}{lcccccccc}
\hline & \multicolumn{3}{c}{ AMBULATÓRIO } & \multicolumn{3}{c}{ INTERNAÇÃO } \\
\hline Idade & \multicolumn{2}{c}{ Sexo M } & \multicolumn{2}{c}{ Sexo F } & \multicolumn{2}{c}{ Sexo M } & \multicolumn{2}{c}{ Sexo F } \\
& N & \% & N & $\%$ & N & $\%$ & N & $\%$ \\
$<30$ anos & - & - & - & - & 1 & 5,9 & - & - \\
31 a 40 anos & 2 & 11,8 & 1 & 5,9 & - & - & - & - \\
41 a 50 anos & - & - & 2 & 11,8 & 4 & 23,5 & 3 & 17,6 \\
51 a 60 anos & 1 & 5,9 & 5 & 29,4 & 1 & 5,9 & 2 & 11,8 \\
61 a 70 anos & 3 & 17,5 & 2 & 11,8 & 3 & 17,6 & 2 & 11,8 \\
71 a 80 anos & - & - & 1 & 5,9 & 1 & 5,9 & - & - \\
TOTAL & 6 & $35,3 \%$ & 11 & $64,7 \%$ & 10 & $58,8 \%$ & 7 & $41,2 \%$ \\
\hline
\end{tabular}


"Em estudos de populações urbanas e em populações hospitalares, tem sido encontrada predominância de mulheres, $66-70 \%$, entre os hipertensos" (BRASIL, 1993a). No entanto, no presente estudo, esta tendência não foi verificada na unidade de internação. No Brasil, considerando dados populacionais de 1985 e a prevalência de HA nas diversas faixas etárias acima de 20 anos de idade, teríamos um total de 8.100 milhões, com $\mathrm{PA}>160 / 95 \mathrm{mmHg}$, sendo que, destes $39 \%$ (3.100 milhões) estão com idade entre 20 e 49 anos. Este valor dobraria se o critério diagnóstico fosse níveis pressóricos > 140/90 mmHg. (BRASIL, 1988).

Segundo BRASIL (1993b), em 1988, as doenças do aparelho circulatório foram responsáveis por $40 \%$ dos bbitos de indivíduos com idade a partir de 45 anos a estimativa de prevalência de HA na população adulta, entre 20 anos de idade ou mais, para 1991 foi de $15 \%$, aproximadamente, 12 milhões de brasileiros. Portanto, "as doenças cardiovasculares representaram a primeira causa de morte da população brasileira e foram responsáveis por, aproximadamente, $34 \%$ dos bbitos do país. Nesse mesmo ano, estimou-se as perdas econômicas no Brasil, número de anos produtivos de vida perdidos, em 1.300 milhões de anos de vida perdidos para essas doenças.
No estado do Rio Grande do Sul, em 1978, foi realizado um estudo com 4.564 indivíduos de 20 a 70 anos revelando que, os níveis médios de PA sistólica elevaram-se consistentemente com a idade em ambos os sexos, essa elevação era maior no sexo feminino, embora iniciassem com níveis mais baixos, acabavam sobrepassando a média dos homens na meia-idade, atingindo níveis bem mais elevado nas mais idosas. Tais diferenças estruturais de sexo e idade são freqüentes e podem levantar questões sobre os seus determinantes genéticos, ambientais e ligados a hábitos de vida (ACHU'T'TI; ACHUTTI, 1997). Outro estudo realizado na cidade de São Paulo, no período de 1988 a 1990 , indicou prevalência menor em mulheres até a idade de 55 anos. Após esta idade, ocorreu um aumento significativo da HA em mulheres (RIBEIRO, 1996). Este fato não foi observado na população deste estudo provavelmente devido a amostragem ter sido não probabilística.

Em relação ao grau de escolaridade (Tabela 2), tanto no ambulatório como na unidade de internação, prevaleceram os indivíduos com o primeiro grau incompleto $(47,1$ e $58,8 \%$, respectivamente). Entretanto, no ambulatório foi elevado o índice de indivíduos sem alfabetização $(29,4 \%)$.

Tabela 2 - Distribuição dos doentes, por unidades de tratamento, quanto o grau de escolaridade. São Paulo, 1997.

\begin{tabular}{lcccc}
\hline & \multicolumn{2}{c}{ AMBULATÓRIO } & \multicolumn{2}{c}{ INTERNAÇÃO } \\
\hline Escolaridade & $\mathbf{N}$ & $\mathbf{N}$ & $\mathbf{N}$ \\
$1^{\circ}$ grau completo & 1 & 5,9 & - & - \\
$1^{\circ}$ grau incompleto & 8 & 47,1 & 10 & 58,8 \\
$2^{\circ}$ grau completo & 3 & 17,6 & 3 & 17,6 \\
$3^{\circ}$ grau completo & - & - & 2 & 11,8 \\
$3^{\circ}$ grau incompleto & - & - & 1 & 5,9 \\
sem alfabetização & 5 & 29,4 & 17 & 5,9 \\
TOTAL & 17 & 100,0 & & 100,0 \\
\hline
\end{tabular}

A escolaridade nos permite avaliar as influências sociais e suas associações aos riscos da HA. Um estudo realizado em Porto Alegre, em 1978, sobre a "prevalência dos fatores de risco" comparou dois extremos da população quanto ao grau de escolaridade. Ficou evidente a maior exposição a todos os fatores de risco, inclusive HA, para os indivíduos sem escolaridade $(27,8 \%)$ do que para aqueles com nível superior (6,6\%)(ACHUTTI; ACHUTTI, 1997). Esta relação inversa entre escolaridade e nível de PA já foi verificada em outros estudos. Indivíduos com $3^{\circ}$ grau completo "tem índice de prevalência de HA 40\% menor que aqueles que têm menos de 10 anos de escolaridade" (BRASIL, 1993a). 
Tabela 3 - Distribuição dos doentes, por unidades de tratamento, quanto a ocupação. São Paulo, 1997.

\begin{tabular}{lcccc}
\hline & \multicolumn{2}{c}{ AMBULATÓRIO } & \multicolumn{2}{c}{ INTERNAÇÃO } \\
\hline Ocupação & $\mathbf{N}$ & $\mathbf{N}$ & $\mathbf{N}$ \\
Prendas do lar & 7 & 41,2 & 3 & 17,6 \\
Aposentado definitivo & 3 & 17,6 & 5 & 29,4 \\
Auxílio doença & 2 & 11,8 & 1 & 5,9 \\
Vínculo empregatício & 2 & 11,8 & 3 & 17,6 \\
Desempregado & 2 & 11,8 & 1 & 5,9 \\
Empregada doméstica & 1 & 5,9 & - & - \\
Autônomo & - & - & $\mathbf{1}$ & 11,8 \\
Pensionista & - & - & 1 & 5,9 \\
Vive de economias & - & - & $\mathbf{1 7}$ & 5,9 \\
TOTAL & 17 & 100,0 & & $\mathbf{1 0 0 , 0}$ \\
\hline
\end{tabular}

Quan to à ocupação verificou-se (Tabela 3) que no ambulatório grande parte dos doentes $(41,2 \%)$ dedica-se a prendas domésticas; já na unidade de internação $29,4 \%$ dos doentes têm aposentadoria definitiva.

As doenças cardiovasculares, além de serem a primeira causa de mortalidade (34\%), representam um enorme ônus financeiro para o país (BRASIL, 1993ab). Segundo o Ministério da Saúde (BRASIL, 1988), em 1983 a HA e suas complicações representavam a terceira causa mais freqüente de auxílios doença concedidos pelo INAMPS $(6,6 \%$ da totalidade de benefícios concedidos/ano) e a primeira causa de invalidez permanente $(20,4 \%$ do total de aposentadorias definitivas concedidas/ano). Ainda, segundo o Ministério da Saúde (BRASIL, 1993a), as doenças do aparelho circulatório referidas acima, corresponderam à cerca de $29 \%$ do total de causa de aposentadorias concedidas pelo INAMPS, entre 1980 e 1986. Neste período, considerando-se o total de causa de aposentadorias definitivas, a HA foi a primeira causa de aposentadoria no país.

Tabela 4 - Distribuição dos doentes, por unidades de tratamento, quanto a renda familiar. São Paulo, 1997.

\begin{tabular}{lcccc}
\hline & \multicolumn{2}{c}{ AMBULATÓRIO } & \multicolumn{2}{c}{ INTERNAÇÃO } \\
\hline Renda familiar & $\mathbf{N}$ & $\%$ & $\mathbf{N}$ & \% \\
Sem salário mínimo & 1 & 5,9 & - & - \\
$<1$ SM$^{*}$ & 1 & 5,9 & - & 11,8 \\
1 a 2 SM & 7 & 41,2 & 2 & 11,8 \\
3 a 4 SM & 2 & 11,7 & 2 & 23,5 \\
5 a 6 SM & 3 & 11,7 & 4 & 52,9 \\
> SM & 3 & 11,7 & 9 & 100,0 \\
TOTAL & 17 & 100,0 & 17 & \\
\hline
\end{tabular}

* Renda em salários mínimos

No ambulatório, 7 doentes $(41,2 \%)$ tinham renda entre 1 e 2 salários, na unidade de internação o quadro se modifica, $52,9 \%$ dos doentes tinham renda maior ou igual a 7 salários, que sustenta 2 a 7 pessoas ou mais (Tabela 4 ).
Dos doentes que referiram possuir moradia propria, $100 \%$ tratavam-se no ambulatório e $88,2 \%$ na unidade de internação. Aqui consideramos como moradia própria também o fato do indivíduo não ter despesas com habitação, como por exemplo aquele que morava com familiares ou em terreno cedido ou 
invadido. Segundo o Ministério da Saúde (BRASIL, 1993a) "as condições sociais de uma população têm peso expressivo na determinação de seu perfil de saúde. Desvantagens no desenvolvimento social e precárias condições de vida acompanham-se, de modo geral, a uma maior risco de adoecer e morrer precocemente, por qualquer causa, em todas as idades, em ambos os sexos". No entanto, são escassos os estudos que relacionam a PA com a organização social ou a maneira como o indivíduo se insere na sociedade.
Quanto ao diagnóstico médico, observou-se (Tabela 5) que no ambulatório a maioria $(88,2 \%)$ dos doentes tem diagnóstico de hipertensão arterial essencial $(47,0 \%)$ ou Sistêmica $(41,2 \%)$. Na unidade de internação o diagnóstico de hipertensão isolada decresce para $41,2 \%$ e aumenta o número de doentes com complicações renais. Estes dados confirmam a prevalência da hipertensão arterial essencial ou primária sobre aquelas conseqüentes às causas secundárias.

Tabela 5 - Distribuição dos doentes, por unidades de tratamento, segundo o diagnóstico médico. São Paulo, 1997.

\begin{tabular}{lcccc}
\hline & \multicolumn{2}{c}{ AMBULATÓRIO } & \multicolumn{2}{c}{ INTERNAÇÃo } \\
\hline Diagnóstico médico & $\mathbf{N}$ & $\mathbf{0}$ & $\mathbf{N}$ & $\%$ \\
Hipertensão Arterial Essencial/Sistêmica & 15 & 88,2 & 7 & 41,2 \\
HA Sistêmica + IRC* & 2 & 11,8 & 5 & 29,4 \\
HA + AVCH** & - & - & 2 & 11,8 \\
HA Secundária/Renovascular & - & - & 1 & 5,9 \\
HA + Renovascular + IRC & - & - & 1 & 5,9 \\
HA + Nefroesclerose benigna & - & - & 1 & 5,9 \\
TOTAL & 17 & 100,0 & 17 & 100,0 \\
\hline
\end{tabular}

* Insuficiência renal crônica.

** Acidente vascular cerebral hemorrágico.

No entanto, "a prevalência de insuficiência renal entre hipertensos é bastante variável em populações diversas pois depende do controle da hipertensão arterial, estando na maioria dos estudos, entre 5 e $15 \%$ dos hipertensos (BRASIL, 1993a). Embora as doenças cardiovasculares representem a terceira causa em número de internações, estão em primeiro lugar em termos de gastos com hospitalizações nos serviços contratados do INAMPS, dados de 1986. A HA é a segunda maior causa cardiovascular de hospitalização $(21,4 \%)$ e representou $2,47 \%$ de todas as internações efetuadas na rede contratada pelo INAMPS, no primeiro semestre de 1987. Portanto, os custos sociais com pacientes hipertensos são elevados, "estima-se cerca de 267 milhões de dólares com consultas médicas, internações, atendimentos de emergência, auxíliodoença, aposentadoria e medicações antihipertensivas". (BRASIL, 1993b).

Tabela 6 - Distribuição dos doentes por unidades de tratamento, e como descobriram a hipertensão arterial. São Paulo, 1997.

\begin{tabular}{lcccc}
\hline & \multicolumn{2}{c}{ AMBULATÓRIO } & \multicolumn{2}{c}{ INTERNAÇÃO } \\
\hline Descobriu a HA: & $\mathbf{N}$ & $\%$ & $\mathbf{N}$ & $\%$ \\
- pela sintomatologia & 5 & 29,4 & 9 & 52,9 \\
- em avaliação médica no trabalho & 1 & 5,9 & 2 & 11,8 \\
- durante a gestação & 5 & 29,4 & 2 & 11,8 \\
- em assistência à saúde por outros agravos & 3 & 17,6 & 3 & 17,6 \\
- em medida ocasional da pressão arterial & 2 & 11,8 & 1 & 5,9 \\
- não lembra & 1 & 5,9 & - & 17 \\
TOTAL & 17 & 100,0 & 100,0 \\
\hline
\end{tabular}


A tabela 6, acima, nos mostra que no ambulatório, 29,4\% referiram terem descoberto a HA através da sintomatologia ou durante a gestação. A maioria $(52,9 \%)$ dos doentes da unidade de internação descobriu ser hipertensos através de sinais e sintomas característicos da doença (mal estar, tontura, cefaléia, cansaço, falta de ar, entre outras).

Quanto a sintomatologia, "as manifestações clínicas da hipertensão arterial sistêmica (HAS) podem ser consideradas enquanto decorrência do estado hipertensivo per se, ou em meio ao complexo sintomatológico de doenças que ocasionalmente determinam a hipertensão arterial ou em meio às manifestações clínicas decorrente de lesões de órgãos determinadas pela hipertensão arterial, ou por seu próprio tratamento" (RIBEIRO, 1996).

Segundo RIBEIRO (1996), comparando com a literatura, a prevalência da descoberta da HA durante a gestação encontramos que aproxi-madamente, $10 \%$ de todas as gestações são complicadas por HA que continua sendo a principal causa de morbi-mortalidade materna e fetal. uma forma convulsiva e grave (eclâmpsia). Estes dados justificam a importância de acompanhamento das mulheres no período pré-natal.

Tabela 7 - Distribuição dos doentes, por unidades de tratamento, e tempo de descoberta da hipertensão arterial. São Paulo, 1997.

\begin{tabular}{lcccc}
\hline & \multicolumn{2}{c}{ AMBULATÓRIO } & \multicolumn{2}{c}{ INTERNAÇÃO } \\
\hline Tempo & $\mathbf{N}$ & $\%$ & $\mathbf{N}$ & $\%$ \\
- há menos de 4 anos & 3 & 17,6 & 7 & 41,2 \\
- entre 10 e 20 anos & 5 & 29,4 & 7 & 41,2 \\
- entre 21 e 30 anos & 6 & 35,3 & 1 & 5,9 \\
- 31 anos ou mais & 3 & 17,6 & 2 & 11,8 \\
TOTAL & 17 & 100,0 & 17 & 100,0 \\
\hline
\end{tabular}

No ambulatório, a maioria dos doentes relata ter história de HA há pelo menos 10 anos. Entretanto, na unidade de internação verificou-se que grande parte $(41,2 \%)$ dos doentes sabem ser hipertensos há menos de 4 anos (Tabela7).

Segundo o Ministério da Saúde (BRASIL, 1993a), "a produção da doença pode ser um processo social influindo ou transformando a base biológica do indivíduo". No entanto, os estudos que associam as variáv eis sociais com a PA são escassos, sendo as variáveis tratadas "independentemente como se fossem características dos individuos, ao invés de reflexos de padrões sociais". Portanto, o conceito de causação da doença deve ser mudado.

Dos doentes entrevistados, tanto no ambulatório quanto na unidade de internação, a metade $(52,9 \%)$ relatou não ter recebido orientação sobre a necessidade de tratamento da HA para sempre; os demais $(47,1 \%)$ disseram terem sido informados a respeito dessa necessidade. Apesar disto, quase a totalidade $(94,1 \%)$ dos hipertensos recebeu algum tipo de orientação sobre o seu tratamento.

Quanto às orientações recebidas pelos hipertensos (Tabela 8), a maioria, tanto no ambulatório (13) quanto na unidade de internação (12) refere ter sido orientada sobre a necessidade de seguir dieta hipossódica. A segunda orientação mais referida foi a tomada da medicação antihipertensiva (10), tanto no ambulatório quanto na unidade de internação (Tabela 8).

Tabela 8 - Distribuição das respostas dos doentes, por unidades de tratamento, quanto às orientações recebidas. São Paulo, 1997.

AMBULATÓRIO

Total de respostas

\section{Orientações}

- dieta hipossódica

- tomar medicação

- parar de ingerir bebidas alcoblicas

- parar de fumar

- diminuir estresse

- diminuir esforço físico/repouso

- fazer exercícios

- diminuir o peso

- comprar aparelho PA

não referiu informação/não lembra
13

10

1

-

2

2

1

-

$-$

INTERNAÇÃO

Total de respostas

12

10

3

4 
O tratamento da HA é para toda a vida. Entretanto, para que o tratamento seja eficiente, é necessário um bom entendimento entre médico e paciente, que leve em conta as preferências de estilo de vida do doente. Além disso, tem como objetivo reduzir ao máximo a $\mathrm{PA}$, até os níveis toleráveis pelo paciente. A HA secundária é tratada de acordo com a sua etiologia enquanto que na $\mathrm{HA}$ essencial, que representa $95 \%$ dos casos de HA, ocorrem mudanças no estilo de vida do paciente (moderação na ingesta de sal, redução de ingesta de álcool e peso, atividade física). Essas mudanças contribuem para diminuir a PA, bem como os fatores de risco associados a $\mathrm{HA}$ (redução do uso de tabaco e lípides). Se os níveis tensionais reduzem, diminuem também a necessidade de medicação anti-hipertensiva. Portanto, essas medidas contribuem para diminuir a incidência da HA, auxiliando na prevenção primária desta patologia. (RIBEIRO; ZANELLA; KOLMANN JR., 1996).
A tabela abaixo (Tabela 9) nos mostra que a maioria dos hipertensos, tanto do ambulatório $(88,2 \%)$ quanto da unidade de internação $(76,5 \%)$, referiu que marcar consulta médica não é uma dificuldade sentida no seguimento do tratamento. É importante lembrar que nesta instituição, os retornos são agendados automaticamente no final de cada consulta médica.Quando questionados se a distância entre a moradia e este serviço de saúde dificultava o tratamento da hipertensão, a maioria $(58,8 \%)$ do ambulatório e 70,6\% da unidade de internação, disse que não. Metade dos doentes tratados no ambulatório justificou que apesar de morar longe da instituição, não sentiu a distância como dificuldade devido a qualidade do atendimento valer o esforço para vir às consultas. Dos $7(41,2 \%)$ doentes do ambulatório que disseram considerar a distância do serviço de saúde como dificuldade para o seguimento do tratamento, 4 justificaram ser por motivos relacionados ao número de conduções e o tempo dispendido nesse transporte.

Tabela 9 - Distribuição dos doentes, por unidades de tratamento, e as dificuldades referidas para o seguimento do tratamento da hipertensão arterial. São Paulo, 1997.

\begin{tabular}{|c|c|c|c|c|c|c|c|c|c|c|c|c|}
\hline \multirow{3}{*}{ Dificuldades } & \multicolumn{5}{|c|}{ AMBULATÓRIO } & \multicolumn{7}{|c|}{ INTERNAÇÃO } \\
\hline & \multicolumn{2}{|c|}{$\operatorname{Sim}$} & \multicolumn{2}{|c|}{ Não } & \multirow[t]{2}{*}{$\mathrm{T}^{*}$} & \multicolumn{2}{|c|}{ Sim } & \multicolumn{2}{|c|}{ Não } & \multicolumn{2}{|c|}{ Não sabe } & $\mathbf{T}^{*}$ \\
\hline & $\mathrm{n}$ & $\%$ & $\mathrm{n}$ & $\%$ & & $\mathrm{n}$ & $\%$ & $\mathrm{n}$ & $\%$ & $\mathrm{n}$ & $\%$ & \\
\hline - marcar consulta médica & 2 & 11,8 & 15 & 88,2 & 17 & 3 & 17,6 & 13 & 76,5 & 1 & 5,9 & 17 \\
\hline - distância até o serviço de saúde & 7 & 41,2 & 10 & 58,8 & 17 & 5 & 29,4 & 12 & 70,6 & - & - & 17 \\
\hline - dinheiro para a compra de medicamentos & 12 & 70,6 & 5 & 29,4 & 17 & 8 & 47,1 & 9 & 52,9 & - & - & 17 \\
\hline - tomar medicamentos & 2 & 11,8 & 15 & 88,2 & 17 & 1 & 5,9 & 16 & 94,1 & - & - & 17 \\
\hline - dinheiro para o transporte & 5 & 29,4 & 12 & 70,6 & 17 & 5 & 29,4 & 12 & 70,6 & - & - & 17 \\
\hline $\begin{array}{l}\text { - horário de atendimento do serviço de } \\
\text { saúde }\end{array}$ & 4 & 23,5 & 13 & 76,5 & 17 & - & - & 16 & 94,1 & 1 & 5,9 & 17 \\
\hline $\begin{array}{l}\text { - demora no atendimento do serviço de } \\
\text { saúde }\end{array}$ & 3 & 17,6 & 14 & 82,4 & 17 & - & - & 16 & 94,1 & 1 & 5,9 & 17 \\
\hline - diminuir o sal da comida & 2 & 11,8 & 15 & 88,2 & 17 & 2 & 11,8 & 15 & 88,2 & - & - & 17 \\
\hline - comer sem sal & 9 & 52,9 & 8 & 47,1 & 17 & 10 & 58,8 & 7 & 41,2 & - & - & 17 \\
\hline
\end{tabular}

* Total

Quanto à dificuldade financeira para comprar medicamentos foi referida por $12(70,6 \%)$ doentes do ambulatório e $8(47,1 \%)$ da unidade de internação. Dos hipertensos internados, $52,9 \%$ disseram que a aquisição de medicamentos não era uma dificuldade. A maioria dos entrevistados, $70,6 \%$ (12) de ambas unidades de tratamento, referiu que o dinheiro para o transporte não se constitui em um problema; 7 desses doentes tratados no ambulatório justificaram esse dado pelo fato de serem idosos ou deficientes físicos, o que os isenta de pagamento de transporte público.
Tomar medicamentos não se constitue em dificuldade para o seguimento do tratamento de $88,2 \%$ dos doentes do ambulatório e de $94,1 \%$ dos doentes da unidade de internação.

A maioria dos entrevistados, $76,5 \%$ do ambulatório e $94,1 \%$ da unidade de internação, referiu não sentir dificuldades em relação ao horário de atendimento do serviço de saúde. O mesmo se deu em relação à demora no atendimento no serviço de saúde, $82,4 \%$ no ambulatório e $94,1 \%$ na unidade de internação. 
Ainda de acordo com a Tabela 9, a maioria dos doentes $88,2 \%$, tanto do ambulatório quanto da unidade de internação, disse não ter dificuldade na diminuição do sal da alimentação. Entretanto, 52,9\% (9) doentes do ambulatório e 58,8\% (10) da unidade de internação disseram ter dificuldade para comer sem sal. Os, 10 hipertensos da unidade de internação, 6 justificaram que a ausência de sal nos alimentos impede a sua ingestão ou é ruim de fazê-la.
A Tabela 10, a seguir, evidenciou que a totalidade dos doentes tratados no ambulatório e a maioria $(88,2 \%)$ daqueles da unidade de internação julgam que receber os medicamentos do serviço de saúde é uma fator facilitante para seguir o tratamento. Outra medida que ajuda no tratamento é a facilidade de marcar consulta nesta instituição, referida por $15(88,2 \%)$ doentes do ambulatório e 14 $(82,4 \%)$ da unidade de internação.

Tabela 10 - Distribuição dos doentes, por unidades de tratamento, e recursos facilitadores para o seguimento do tratamento da hipertensão arterial. São Paulo, 1997.

\begin{tabular}{|c|c|c|c|c|c|c|c|c|c|c|c|c|}
\hline \multirow{3}{*}{ Facilidades } & \multicolumn{5}{|c|}{ AMBULATÓRIO } & \multicolumn{7}{|c|}{ INTERNAÇÃo } \\
\hline & \multicolumn{2}{|c|}{ Sim } & \multicolumn{2}{|c|}{ Não } & \multirow[t]{2}{*}{$T^{*}$} & \multicolumn{2}{|c|}{ Sim } & \multicolumn{2}{|c|}{ Não } & \multicolumn{2}{|c|}{ Não sabe } & \multirow[t]{2}{*}{$\mathrm{T}^{*}$} \\
\hline & $\mathrm{n}$ & $\%$ & $\mathrm{n}$ & $\%$ & & $\mathrm{n}$ & $\%$ & $\mathrm{n}$ & $\%$ & $\mathrm{n}$ & $\%$ & \\
\hline - receber os medicamentos do serviço de saúde & 17 & 100 & - & - & 17 & 15 & 88,2 & 2 & 11,8 & - & - & 17 \\
\hline - facilidade de marcar consulta & 15 & 88,2 & 2 & 11,8 & 17 & 14 & 82,4 & 3 & 17,6 & - & - & 17 \\
\hline - proximidade do serviço de saúde & 13 & 76,5 & 4 & 23,5 & 17 & 12 & 70,6 & 4 & 23,5 & 1 & 5,9 & 17 \\
\hline - vale transporte pelo Serv. Assistência Social & 12 & 70,6 & 5 & 29,4 & 17 & 8 & 47,1 & 9 & 52,4 & - & - & 17 \\
\hline - apoio familiar & 12 & 70,6 & 5 & 29,4 & 17 & 15 & 88,2 & 2 & 11,8 & - & - & 17 \\
\hline
\end{tabular}

* Total

A maioria dos hipertensos do ambulatório (76,5\%) e da unidade de internação $(70,6 \%)$ consideram a proximidade do serviço de saúde como um facilitador para o seguimento do tratamento; $70,6 \%$ dos doentes do ambulatório e $47,1 \%$ da unidade de internação, referiram que o fornecimento de vale transporte pelo serviço de assistência social se constituiria em facilitador para o tratamento. Já $52,4 \%$ dos hipertensos da unidade de internação relataram que esta medida não se faz necessária para o seu tratamento mas que poderia facilitar o tratamento daqueles que precisam da ajuda financeira para o transporte. $70,6 \%$ dos doentes do ambulatório e $88,2 \%$ da unidade de internação consideram ser importante o apoio familiar para o seguimento do tratamento da HA.

\section{CONSIDERAÇÕES FINAIS}

Este estudo realizado com 34 doentes, sendo 17 em tratamento ambulatorial e 17 em tratamento na unidade de internação de nefrologia permitiu as considerações finais descritas a seguir.

\subsection{Quanto à caracterização da clientela estudada}

- No ambulatório: predominou o sexo feminino, que dedica-se à prendas domésticas, acometida por HA Essencial, com idade entre 51 e 60 anos e escolaridade de 1(grau incompleto; para o sustento de 1 a 4 pessoas têm renda familiar entre 1 e 2 salários mínimos.
- Na unidade de internação: predominou a clientela do sexo masculino, com aposentadoria definitiv a e diagnóstico de HA Essencial, isolada ou já com suas complicações renais; idade entre 41 a 50 anos, escolarização de 1 ( grau incompleto e renda familiar maior ou igual a 7 salários mínimos.

\subsection{Quanto ao conhecimento do diagnóstico e o seguimento do tratamento}

- No ambulatório: os doentes referem a descoberta da doença predominantemente através da sintomatologia ou durante a gestação, sendo que a maioria relata ter história de HA há pelo menos 10 anos e, metade diz não saber que o tratamento seria para sempre; as orientações recebidas para o controle da doença englobaram principalmente a tomada da medicação e a ingestão de dieta hipossódica; em relação ao seguimento do tratamento as dificuldades mais referidas para esse seguimento foram: dinheiro para a compra de medicamentos, comer alimentos sem sal, distância até o serviço de saúde e dinheiro para o transporte; quanto aos recursos facilitadores do tratamento a totalidade da clientela aponta o recebimento gratuito de medicamentos da instituição e, a maioria julga que a facilidade de marcar consultas, a proximidade do serviço, o fornecimento de vale transporte e o apoio familiar ajudariam no seguimen to do tratamento.

- Na unidade de internação: grande parte dos doentes descobriu ser hipertenso através da 
sintomatologia, há menos de 4 anos ou entre 10 e 20 anos; e, desconhecia dever tratar-se para sempre; as orientações recebidas para o controle da HA envolveram principalmente a tomada de medicação e a ingestão de dieta hipossódica; quanto às dificuldades para o seguimento do tratamento, foram apontadas a ingestão de dieta hipossódica, o dinheiro para a compra de medicamentos e para o transporte e, a distância até o serviço de saúde; em relação aos recursos facilitadores, a maioria referiu o recebimento gratuito de medicamentos da instituição, o apoio familiar, a facilidade de marcar consultas e a proximidade do serviço de saúde como aqueles que mais ajudariam no tratamento.

Em geral não houve diferenças entre as dificuldades e as facilidades referidas pelos doentes para o seguimento do tratamento da HA nos dois momentos estudados. As dificuldades e facilidades apontadas pelos doentes referem-se ao financiamento do tratamento (compra de medicamento e gastos com transporte); acessibilidade ao serviço (facilidade em marcar consulta médica e distância do serviço de saúde); mudança de hábito alimentar (dieta hipossódica) e a importância do apoio familiar nesta trajetória.

\section{REFERÊNCIAS BIBLIOGRÁFICAS}

ACHUTTI, A.C.; ACHUTTI, V.A.R. Aspectos epidemiológicos. In: AMODEO, C.; LIMA, E.G.; VAZQUEZ, E.C. (coord.) Hipertensão arterial. Sao Paulo, Sarvier, 1997, cap. 2, p. 11-21.

BLACK, J.M.; MATASSARIN-JACOBS, E. Luckmann \& Sorensen. Enfermagem médico-cirúrgica: uma abordagem psicofisiológica. 4 ed. Rio de Janeiro, Guanabara Koogan, 1993. cap. 9, p. 117-33: Condiçбes crônicas.

BRASIL. Ministério da Saúde. Secretaria Nacional de Programas Especiais de Saúde. Divisão Nacional de Doenças Crônico-Degenerativas. Programa Nacional de Educação e Controle da Hipertensão Arterial. Normas técnicas para o Programa Nacional de Educação e Controle da Hipertensão Arterial. Brasília, Centro de Documentação do Ministério da Saúde, 1988 p. 7-53.

BRASIL. Ministério da Saúde. Secretaria de Assistência à Saúde. Departamento de Programas de Saúde. Coordenação de Doenças Cardio-vasculares. Controle de hipertensao arterial: uma proposta de integraçăo ensino-serviço. Rio de Janeiro, CDCV/NUTES, 1993a.
BRASIL. Ministério da Saúde. Secretaria de Assistência à Saúde. Departamento de Programas de Saúde. Coordenação de Doenças Cardiovasculares. Doenças cardiovasculares no Brasil, Sistema Único de Saúde - SUS: dados epidemiológicos e assistência médica. Brasília, 1993b. p.9-35.

CARVALHO, J.G.R de. Rim e hipertensão arterial. In: AMODEO, C.; LIMA, E.G.; VAZQUEZ, E.C. (coord.) Hipertensão arterial. São Paulo, Sarvier, 1997. cap. 18, p. 141-52.

COSTA, C.A.R. Hipertensão arterial sistêmica do adulto. In: BARROS, E. et al. Nefrologia: rotinas, diagnóstico e tratamento. Porto Alegre, Artes Médicas Sul, 1994. cap. 5, p. 49-54

CONSENSO BRASILEIRO DE HIPERTENSÃO ARTERIAL, 3 , Campos do Jordão, 1988. Anais, Campos do Jordão, Sociedade Brasileira de Hipertensao, 1988

FLEURY, S.; GIOVANELLA, L. Universalidade da atenção à saúde: acesso como categoria de análise. In: CIBENSCHUTZ E. (org.) Política de saúde: o público e o privado. Rio de Janeiro, Fiocruz, 1996. p. 177-99.

DELITTI, A.M. de C.; SILVA, D. da. Relação médico-pacientehipertensão: um estudo psicossociológico. São Paulo, Limay, 1996.

PASCOAL, I.F.; MION JR. D. Hipertensão arterial e o rim. In: RIBEIRO, A.B. (org.) Atualização em hipertensão arterial clínica, diagnóstico e terapêutica. São Paulo, Atheneu, 1996. cap. 10, p.97-108.

PIERIN, A.M.G. et al. Atendimento de enfermagem ao paciente com hipertensão arterial. Rev. Bras.Med.Card., v.3, n.4 p. $209-11,1984$

RIBEIRO, M.B.D. Epidemiologia da hipertensão arterial. In RIBEIRO, A.B. (org.) Atualização em hipertensão arterial: clínica, diagnóstico e terapêutica. Såo Paulo, Atheneu, 1996. cap. 2 , p.9.19.

RIBEIRO, A.B.; ZANELLA, M.T.; KOHLMANN JUNIOR, O Tratamento da hipertensão arterial. Atualização em hipertensão arterial: clínica, diagnóstico e terapêutica. São Paulo, Atheneu, 1996. cap. 18, p.193-223.

THOMÉ, F.S. Prevenção de doenças renais. In: BARROS, E. et al. Nefrologia: rotinas, diagn6stico e tratamento. Porto Alegre, Artes Médicas Sul, 1994. cap. 1, p.1-10.

TOTO, R.D. et al. "Strict" blood pressure control and progression of renal disease in hypertensive nephrosclerosis. Kidney International. v.48, n. 3, p.851-9, 1995. 


\section{ANEXO}

Parte I -Identificação e Condições S6́cio-Econômicas

1. Registro $\mathrm{HC} \mathrm{N}$ :

2. Entrevista $\mathrm{N}^{\circ}$ :

3. Serviço: ( ) Ambulatório ( ) Internação

4. Diagnóstico:

5. Sexo: ( ) F

( ) $\mathrm{M}$

6. Idade: anos

7. Já ficou internado (a) alguma vez internado devido a hipertensão e ou suas complicações? ( ) S ( ) N

8. Escolaridade: $-1^{\circ} \mathrm{g} \mathrm{C} \mathrm{(} \mathrm{)} \mathrm{I} \mathrm{(} \mathrm{)}-2^{\circ} \mathrm{g} \mathrm{C} \mathrm{(} \mathrm{)} \mathrm{I} \mathrm{(} \mathrm{)} \mathrm{-} 3^{\circ} \mathrm{g} \mathrm{C}($ ) I ( ) - sem alfab ( )

9. Ocupação: - com vínculo empregatício $\mathrm{S}($ ) $\mathrm{N}($ ) - aposentado definitivo ( ) - auxílio doença ( ) - pensionista ( ) - prendas do lar ( ) - empregada doméstica ( ) - outros?

10. Renda familiar: - < $1 \mathrm{SM}($ ) - 1 e $2 \mathrm{SM}($ ) -3 e $4 \mathrm{SM}($ ) -5 e $6 \mathrm{SM}($ ) - ( $7 \mathrm{SM}($ )

11. Número de pessoas da família que vivem com esta renda?

12. Moradia: - própria ( ) - alugada ( ) - cedida/emprestada ( ) - outro. Qual?

Parte II - Dados sobre Seguimento do Tratamento da Hipertensão Arterial

13. Como o (a) Sr. (a) descobriu ser hipertenso?

14. Há quanto tempo sabe ser hipertenso?

15. No momento em que descobriu ser hipertenso, recebeu alguma orientação sobre a necessidade de tratar a hipertensão para sempre? S( ) N ( )

16. Quais orientações recebeu no momento em que descobriu ser hipertenso?

17. O que dificulta o (a) Sr. (a) a seguir o tratamento da hipertensão?

( ) marcar consulta médica

( ) dinheiro para a compra de medicamentos

( ) tomar os medicamentos

( ) dinheiro para o transporte

( ) horário de atendimento do serviço de saúde

( ) demora no atendimento do serviço de saúde

( ) diminuir o sal da comida

( ) distância até o serviço de saúde

( ) comer sem sal

18. O que ajuda o Sr.(a) a seguir o tratamento da pressão alta?

( ) receber os medicamentos do serviço de saúde

( ) facilidade de marcar consulta médica

( ) proximidade do serviço de saúde (da moradia/emprego/outro)

( ) fornecimento de vale transporte pelo serviço de assistência social

( ) apoio familiar 